\title{
Tyrosinase-related protein 1 mRNA expression in lymph node metastases predicts overall survival in high-risk melanoma patients
}

P El Hajj ${ }^{1,7}$, F Journe ${ }^{1,7}$, M Wiedig ${ }^{1}$, I Laios ${ }^{2}$, F Salès ${ }^{1}$, M-D Galibert ${ }^{3}$, LC Van Kempen ${ }^{4}$, A Spatz ${ }^{4}$, B Badran ${ }^{5}$, D Larsimont ${ }^{2}$, A Awada ${ }^{6}$ and G Ghanem*,1

${ }^{1}$ Laboratoire d'Oncologie et de Chirurgie Expérimentale, Institut Jules Bordet, Université Libre de Bruxelles, B1000 Brussels, Belgium; ${ }^{2}$ Service d'Anatomie Pathologique, Cytologie et Cytogénétique, Institut Jules Bordet, Université Libre de Bruxelles, Brussels, Belgium; ${ }^{3}$ CNRS UMR 6061, Institut de Génétique et Développement de Rennes, Rennes, France; ${ }^{4}$ Department of Pathology, McGill University and Lady Davis Institute for Medical Research, Jewish General Hospital, Montreal, QC, Canada; ${ }^{5}$ Department of Biochemistry, Laboratory of Immunology, Faculty of Sciences, Lebanese University, Hadath-Beirut, Lebanon and ${ }^{6}$ Clinique d'Oncologie Médicale, Institut Jules Bordet, Université Libre de Bruxelles, Brussels, Belgium

Background: Clinical outcome of high-risk melanoma patients is not reliably predicted from histopathological analyses of primary tumours and is often adjusted during disease progression. Our study aimed at extending our previous findings in skin metastases to evaluate the prognostic value of tyrosinase-related protein 1 (TYRP1) in lymph node metastases of stages III and IV melanoma patients.

Methods: TYRP1 mRNA expression in 104 lymph node metastases was quantified by real-time PCR and normalised to S100 calcium-binding protein B (S100B) mRNA expression to correct for tumour load. TYRP1/S100B ratios were calculated and median was used as cutoff value. TYRP1/S100B mRNA values were correlated to clinical follow-up and histopathological characteristics of the primary lesion.

Results: A high TYRP1/S100B mRNA ratio significantly correlated with reduced disease-free (DFS) and overall survival (OS; Cox regression analysis, $P=0.005$ and 0.01, respectively), increased Breslow thickness (Spearman's rho test, $P<0.001$ ) and the presence of ulceration (Mann-Whitney test, $P=0.02$ ) of the primaries. Moreover, high TYRP1/S100B was of better prognostic value (lower $P$ value) for OS than Breslow thickness and ulceration. Finally, it was well conserved during disease progression with respect to high/ low TYRP1 groups.

Conclusion: High TYRP1/S100B mRNA expression in lymph node metastases from melanoma patients is associated with unfavourable clinical outcome. Its evaluation in lymph node metastases may refine initial prognosis for metastatic patients, may define prognosis for those with unknown or non-evaluable primary lesions and may allow different management of the two groups of patients.

\footnotetext{
*Correspondence: G Ghanem; E-mail: gghanem@ulb.ac.be

${ }^{7}$ These authors contributed equally to this paper.
}

Received 13 November 2012; revised 20 February 2013; accepted 21 February 2013; published online 21 March 2013

(c) 2013 Cancer Research UK. All rights reserved 0007 - 0920/13 
Cutaneous melanoma is the less common (4\%) and the deadliest form of skin cancer; it causes the majority of deaths related to skin cancer (75\%), with an increasing incidence in the last decades (Berwick et al, 2009). Early detection and complete surgical excision of melanoma are the best strategies to reduce mortality. However, about $15 \%$ of patients diagnosed with primary melanoma develop distant metastases. Treatment options for advanced melanoma are limited and rarely curative with survival rates often below 15\% (Miller and Mihm, 2006). Importantly, although long-term survival for patients with advanced melanoma is low, it is highly variable (Balch et al, 2010). Many efforts are done to identify metastatic melanoma patients with a longer life expectancy. Thus, it would potentially be useful to classify melanoma that has already metastasised into categories that more accurately predict patient survival. Indeed, in the absence of effective therapy for the late-stage melanoma, the early identification of patients of better prognostic is critical.

The current melanoma AJCC staging system proposed clinical and pathological prognostic factors to estimate the survival of melanoma patients (Balch et al, 2009). However, a recent multivariate analysis of prognostic factors among 2313 patients with stage III melanoma demonstrated remarkable heterogeneity of prognosis (Balch et al, 2010). Such results support the evaluation of additional marker(s) in metastases of melanoma patients in order to refine the initial prognosis and reclassify patients at the time of melanoma progression. In this context, various groups identified genes associated with survival (Mandruzzato et al, 2006) or suggested the use of serum and tissue markers in order to refine the prognosis of patients with high-risk melanoma, to ensure adequate follow-up, and to predict the possible benefit from a therapy. These markers, associated with a significant poorer prognosis and a shorter survival, included high level of the autophagosome marker LC3B (Lazova et al, 2012), elevated cytoplasmic p27 levels (Chen et al, 2011), high p-proteasome levels (Henry et al, 2010), both elevated S100B and MIA (melanoma inhibitory activity; Díaz-Lagares et al, 2011), high vascular endothelial growth factor-C and its receptor VEGFR-3 (Mouawad et al, 2009), high percentage of protease inhibitor 9positive tumour cells (van Houdt et al, 2005), strong nuclear ATF2 expression (Berger et al, 2003), high L-Dopa/L-Tyrosine ratio and high Ki-67-labeling index (Stoitchkov et al, 2003). However, most studies consist in small series requiring additional evaluation in larger populations and thus, their results have not been translated into a meaningful prognostic tool in clinics up-to-now.

In our previous study combining microarray analysis and realtime PCR in skin melanoma metastases, we found significant correlation between tyrosinase-related protein 1 (TYRP1) expression and distant metastasis-free survival, overall survival (OS) and Breslow thickness, suggesting that TYRP1 can be of a prognostic value particularly useful when pathology parameters at the primary lesion are lacking (about 20\% of all cases of both unknown and some ulcerated primaries; Journe et al, 2011).

In the current study, we extend our initial observations by evaluating TYRP1 mRNA expression in 104 lymph node metastases from melanoma patients by real-time PCR. As lymph node biopsies may contain variable amounts of stroma and tumour tissue, we related TYRP1 values to those of S100 calciumbinding protein $\mathrm{B}(\mathrm{S} 100 \mathrm{~B})$ mRNA, a gene whose expression is specific of melanocyte lineage, thus accounting for tumour load. We calculated correlations between TYRP1/S100B mRNA expression and disease-free survival (DFS), OS and conventional histopathological parameters, such as Breslow thickness, ulceration and lymph node involvement. Then, we compared the prognostic value of TYRP1/S100B to those of Breslow thickness and ulceration.

\section{MATERIALS AND METHODS}

Patients and tissue collection. Lymph node metastases were collected from patients with stage III $(n=86)$ and IV $(n=18)$ melanoma undergoing surgery as a part of the diagnostic work-up or therapeutic strategy at Institut Jules Bordet (Brussels, Belgium) from 1998 to 2009. Samples were collected randomly with no inclusion or exclusion criteria. All were palpable lymph node macrometastases confirmed by sonography and pathology examination. Immediately after surgery, specimens were snap-frozen in liquid nitrogen and stored at $-80{ }^{\circ} \mathrm{C}$ until use. This study was approved by the ethic committee of Institut Jules Bordet and performed in accordance with the REMARK guidelines (Alonzo, 2005; McShane et al, 2005). The clinical characteristics of the patients are outlined in Table 1.

RNA extraction. Frozen samples were homogenised using the FastPrep-24 homogeniser system with lysing matrix D (MP Biomedicals, Illkirch, France) in RLT buffer supplemented with $\beta$-mercaptoethanol (RNeasy Mini Kit, Qiagen, Venlo, The Netherlands) at $4{ }^{\circ} \mathrm{C}$, and then centrifuged with RNeasy spin column for the separation of melanin from the total RNA. After washing steps, RNA was collected in RNase-free water and subjected to DNase treatment as described by the manufacturer. RNA concentration was evaluated using a NanoDrop 1000 spectrophotometer (Thermo Scientific, Wilmington, DE, USA). RNA quality was assessed based on the RNA profile generated by a Bioanalyzer 2100 (Agilent Technologies, Santa Clara, CA, USA); all samples used in this study had RNA integrity number values $>6$.

Real-time PCR. TYRP1, S100B and $\beta$-actin mRNA expression were quantified by real-time PCR. cDNA was synthesised using a standard reverse transcription method (qScript cDNA SuperMix, Quanta Biosciences, Gaithersburg, MD, USA). Real-time PCR reactions were performed using the SYBR Green PCR Master Mix (Applied Biosystems, Foster City, CA, USA) and sequence-specific primer sets designed from PrimerBank (http://pga.mgh.harvard. edu/primerbank/) for TYRP1 (forward: 5'-AGCCCTCAGTATCC CCATGAT-3', reverse: 5'-CCCGGACAAAGTGGTTCTTTT-3', amplicon size: 214), for S100B (forward: 5'-TGGCCCTCATCGAC GTTTTC-3', reverse: 5'-ATGTTCAAAGAACTCGTGGCA-3', amplicon size: 248 ) and for $\beta$-actin (forward: $5^{\prime}$-CATGTACGTT GCTATCCAGGC-3', reverse: $5^{\prime}$-CTCCTTAATGTCACGCACG AT-3', amplicon size: 250; Life Technologies, Gent, Belgium). The amplification was performed on a LightCycler 480 System (Roche Diagnostics GmbH, Mannheim, Germany) using an initial activation step $\left(95^{\circ} \mathrm{C}\right.$ for $\left.10 \mathrm{~min}\right)$ followed by 40 cycles of amplification $\left(95^{\circ} \mathrm{C}\right.$ for $15 \mathrm{~s}$ and $60{ }^{\circ} \mathrm{C}$ for $\left.60 \mathrm{~s}\right)$. Melting curves from $60{ }^{\circ} \mathrm{C}$ to $99^{\circ} \mathrm{C}$ were assessed to evaluate PCR specificity. A preliminary analysis demonstrated linear and similar amplification efficacies. Relative quantification was determined by normalising the crossing threshold (CT) of TYRP1 and S100B with the CT of $\beta$-actin (loading control) using the $2^{-\Delta \mathrm{CT}}$ method. Both relative mRNA expressions were then used to calculate TYRP1/S100B ratio.

Statistical analysis. Statistical correlation between two variables was assessed using Spearman's rho test. Statistical significance between two independent groups was examined using the MannWhitney test. Disease-free survival and OS were estimated using the Kaplan-Meier method. Univariate and multivariate analyses were performed by Cox regression model to estimate hazard ratios (HRs) and 95\% confidence intervals. Significance of the positive predictive value was determined by Fisher's exact test. $P$-values $<0.05$ were considered as statistically significant. All statistical analyses were performed using SPSS 15.0 Inc. (Chicago, IL, USA). 


\begin{tabular}{|c|c|c|c|}
\hline & Median (range) & $\begin{array}{l}\text { Number of } \\
\text { samples in } \\
\text { category }\end{array}$ & $\begin{array}{l}\text { Frequency } \\
\text { (\%) }\end{array}$ \\
\hline Histology of primary & & 104 & \\
\hline Unknown & & 14 & 13.5 \\
\hline Unclassable & & 10 & 9.6 \\
\hline SSM & & 46 & 44.2 \\
\hline NM & & 20 & 19.2 \\
\hline ALM & & 8 & 7.7 \\
\hline LMM & & 1 & 1.0 \\
\hline Mucosal & & 5 & 4.8 \\
\hline Gender & & 104 & \\
\hline Male & & 47 & 45.2 \\
\hline Female & & 57 & 54.8 \\
\hline Age (years) ${ }^{a}$ & $52(25-87)$ & 104 & \\
\hline $15-39$ & & 26 & 25.0 \\
\hline $40-64$ & & 49 & 47.1 \\
\hline$\geqslant 65$ & & 29 & 27.9 \\
\hline \multicolumn{4}{|l|}{ Survival data (years) } \\
\hline $\mathrm{DFS}^{\mathrm{b}}$ & $1.2(0.1-25.7)$ & 104 & \\
\hline $\mathrm{OS}^{\mathrm{c}}$ & $5.1(0.8-29.3)$ & 100 & \\
\hline Breslow $^{d}$ & $2.2(0.7-45.0)$ & 94 & \\
\hline$\leq 1.00$ & & 10 & 10.6 \\
\hline $1.01-2.00$ & & 30 & 31.9 \\
\hline $2.01-4.00$ & & 39 & 41.5 \\
\hline$>4.00$ & & 15 & 16.0 \\
\hline Ulceration $^{\mathbf{e}}$ & & 54 & \\
\hline Yes & & 33 & 61.1 \\
\hline No & & 21 & 38.9 \\
\hline $\begin{array}{l}\text { Lymph node } \\
\text { involvement }^{f}\end{array}$ & & 79 & \\
\hline 0 & & 50 & 63.3 \\
\hline$\geqslant 1$ & & 29 & 36.7 \\
\hline Treatment & & 74 & 71.2 \\
\hline Chemotherapy & & 32 & 30.8 \\
\hline Immunotherapy & & 20 & 19.2 \\
\hline Chemo/immunotherapy & & 22 & 21.2 \\
\hline No treatment & & 30 & 28.8 \\
\hline TYRP1/S100B & $\begin{array}{c}8.7 \times 10^{-3} \\
\left(5.2 \times 10^{-6}-63.6\right) \\
\end{array}$ & 104 & \\
\hline \multicolumn{4}{|c|}{ 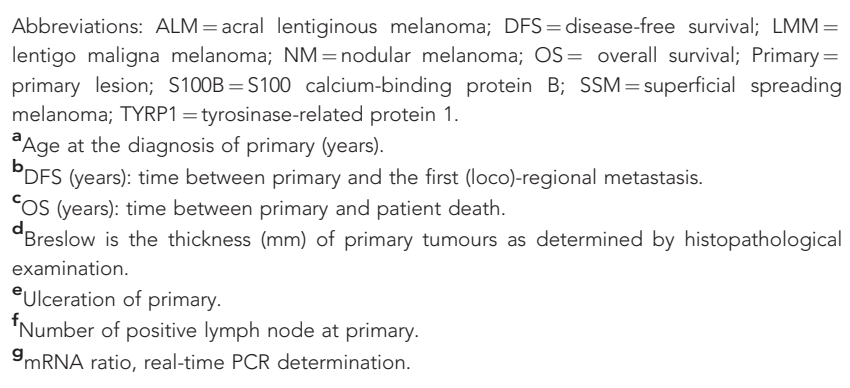 } \\
\hline
\end{tabular}

\section{RESULTS}

Characteristics of melanoma patients. The clinical and pathological characteristics of melanoma patients whose lymph node metastases have been evaluated in this study are reported in Table 1. Samples were obtained from patients diagnosed between 1981 and 2007. The majority of the melanomas were of superficial spreading or nodular histological subtypes. Eighty-nine percent of patients had primary melanoma with Breslow thickness $>1 \mathrm{~mm}$. Data for ulceration were known for about half of the patients (52\%). Disease-free survival and OS ranged from 0.1 to 25.7 and from 0.8 to 29.3 years with median of 1.2 and 5.1 years, respectively. Of note, OS refers to disease-specific survival. Four patients were lost for follow-up for OS. In all, $81 \%$ of patients were deceased at the time of the study, 19\% were still alive. About onethird of patients were only treated by surgery, whereas other received additional chemotherapy (DTIC, cisplatin, etc.), immunotherapy (interferon $\alpha$-2b or vaccine) or both therapies (Table 1 ). These treatments did not affect OS (Mann-Whitney test, $P>0.270$ ). No patient included in the present study has been treated with the recent anti-CTLA4 antibodies or the BRAF inhibitors.

Evaluation of TYRP1/S100B ratio in lymph node metastases. To rule out any possible bias due to variations in tumour tissue content of lymph node samples, we calculated the specific melanocyte marker $\mathrm{S} 100 \mathrm{~B} / \beta$-actin ratio and corrected the TYRP $1 / \beta$-actin value by calculating a TYRP1/S100B ratio (Table 1). Of note, $S 100 \mathrm{~B} / \beta$-actin ranged from 0.001 to 8.815 (median $=0.226$ ) confirming a high variation in tumour load among lymph node samples. In addition, we checked that TYRP1/ $\beta$-actin did not correlate with $\mathrm{S} 100 \mathrm{~B} / \beta$-actin $(r=0.050, P=0.615$, Spearman's rho test), suggesting no direct relationship between the expression of both genes.

Correlation of TYRP1/S100B ratio with pathological features of the primary lesion. TYRP1/S100B mRNA expression (as a continuous variable) in lymph node metastases significantly correlated with Breslow thickness $(P<0.001)$ and ulceration $(P=0.023)$ of the primary lesions of melanoma patients (Table 2), demonstrated that TYRP1/S100B is not an independent factor. However, it did not correlate with the number of positive lymph nodes detected at the time of excision of the primary $(P=0.504)$. Moreover, we also found significant correlations between TYRP1/S100B ratio and both DFS $(P=0.017)$ and OS $(P=0.008 ;$ Table 2$)$. Of note, although TYRP $1 / \beta$-actin showed excellent correlation with TYRP1/S100B $(\rho=0.888, P<0.001$, Spearman's rho test) and OS $(\rho=-279, P=0.005)$, it did not significantly correlate with DFS $(P=0.123)$, supporting the fact that normalisation of TYRP1 with S100B improved significance. Of note, $\mathrm{S} 100 \mathrm{~B} / \beta$-actin did not correlate with neither DFS $(P=0.07$, Cox regression analysis) nor OS $(P=0.39)$.

Comparison of the prognostic values of TYRP1/S100B ratio, Breslow thickness and ulceration. To find out the most relevant cutoff point for TYRP1/S100B ratio, we divided the population into two groups according to the median, the 25th and the 75th percentiles. We found that the median cutoff point gave a lower $P$-value than other cutoff points with a comparable HR for DFS and OS (Cox regression analysis; Figure 1). Then, using the median cutoff point, we defined a group with 'low TYRP1' and a group with 'high TYRP1' and showed the corresponding Kaplan-Meier curves in Figure 2 (top panel). High TYRP1 was significantly associated with a shorter DFS and a shorter OS in univariate analyses.

The prognostic power of TYRP1/S100B ratio was then compared with those of Breslow and ulceration by considering a median Breslow thickness of $2.3 \mathrm{~mm}$ and the presence/absence of ulceration as cutoff points. Kaplan-Meier analyses and Cox regressions showed that high Breslow thickness was significantly correlated with a shorter DFS and a shorter OS (Figure 2, medium panel). Similarly, ulceration of primaries was also significantly associated with a shorter DFS and tended to be significant with a shorter OS $(P=0.058)$ (Figure 2 , bottom panel). Altogether, comparing $P$-values and HRs for TYRP1, Breslow and 
Table 2. Association of TYRP1 mRNA expression in lymph node metastases with pathological parameters of primaries

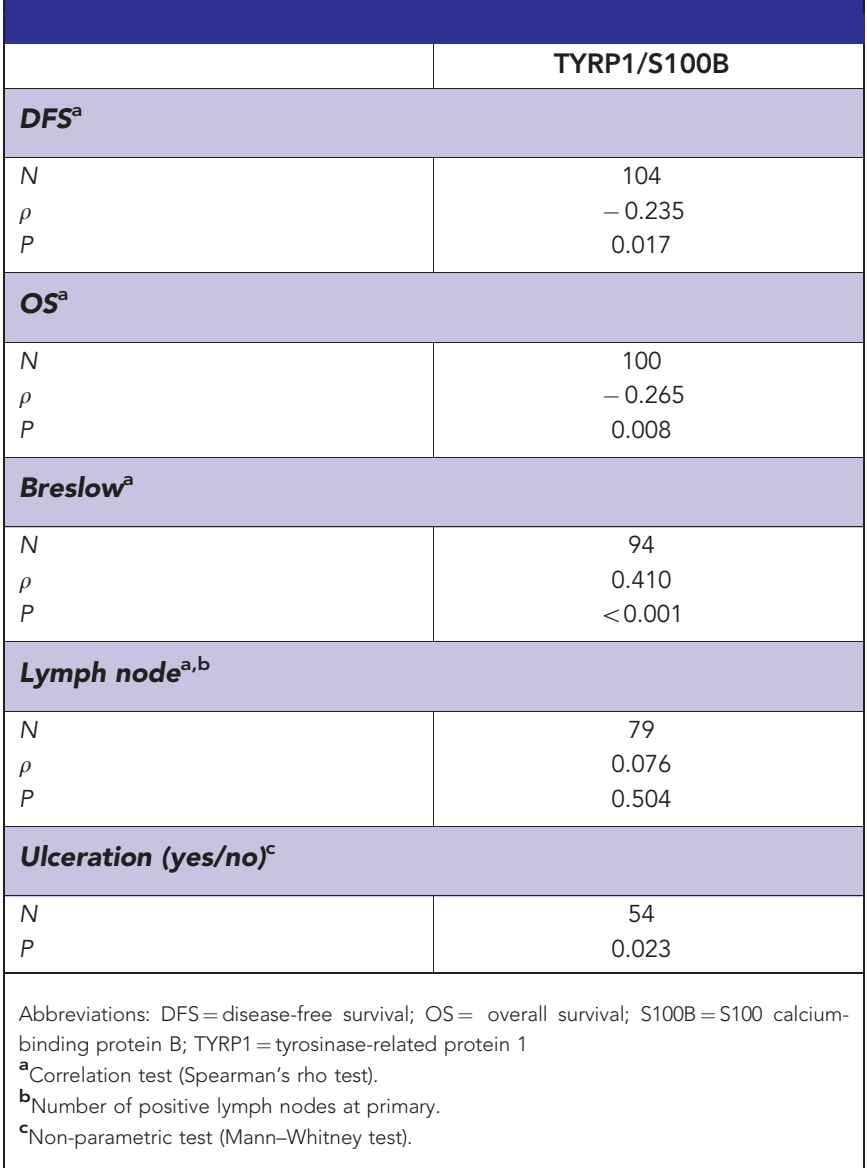
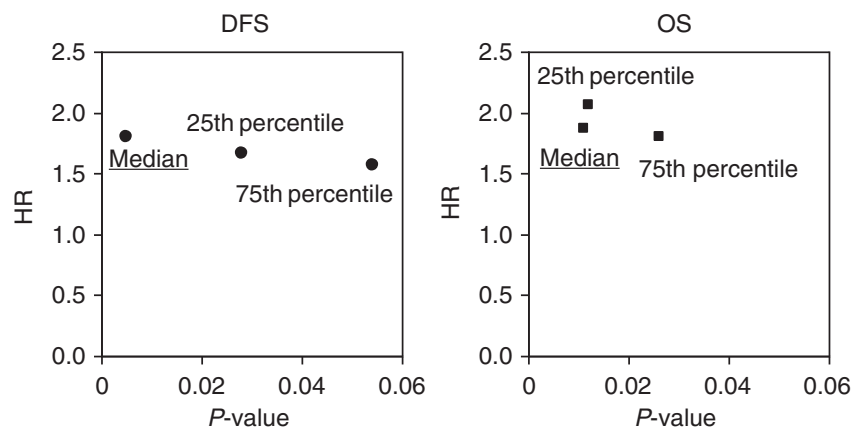

Figure 1. Identification of a cutoff point for TYRP1/S100B ratio. Population $(n=104)$ was classified with regard to ascending TYRP1/ $\mathrm{S} 100 \mathrm{~B}$ ratio that was divided into two groups according to the median, the 25 th percentile and the 75 th percentile. $P$-values and hazard ratios (HR) were calculated (Cox regression) for DFS and OS for each cutoff point.

ulceration, we found that all markers gave similar HRs but Breslow thickness showed a lower $P$-value for DFS and TYRP1 demonstrated a lower $P$-value for OS (Figure 3 ). These data suggest that TYRP1 could be a more valuable marker to refine prognosis of metastatic patients.

Moreover, high TYRP1, high Breslow and ulceration predicted, respectively, 88\% $(P=0.049$, Fisher's exact test $), 94 \%(P=0.003)$ and $100 \%(P=0.006)$ of first relapse before 5 years and $82 \%$ $(P=0.003), 81 \%(P=0.007)$ and $91 \%(P=0.035)$ of mortality before 10 years. Globally, TYRP1 evaluated in lymph node
TYRP1/S100B
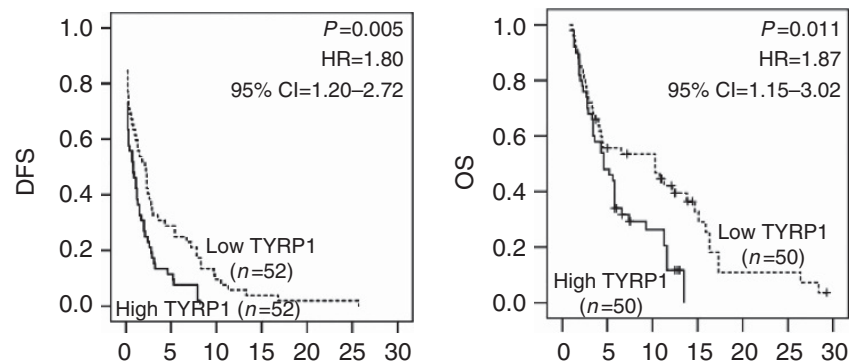

Breslow
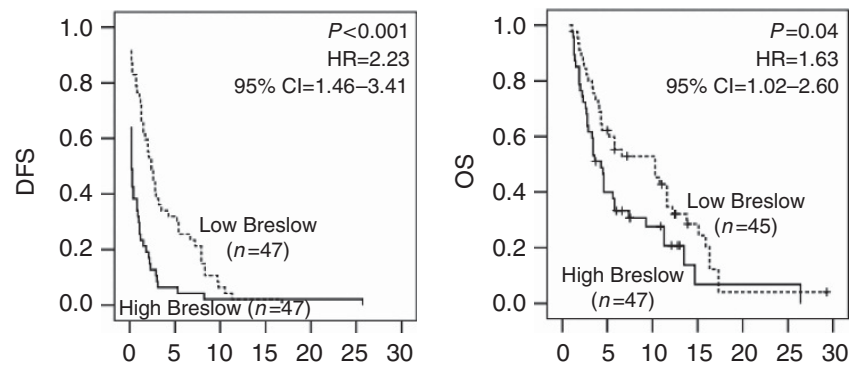

Ulceration
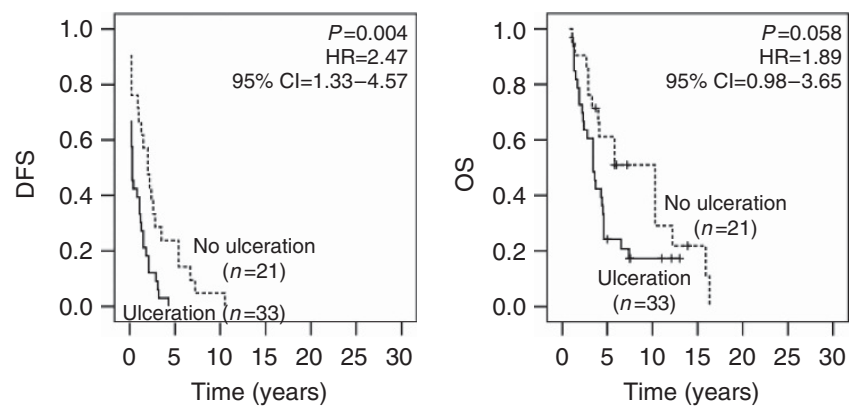

Figure 2. Survival curves for TYRP1/S100B ratio, Breslow and ulceration. DFS and OS curves (Kaplan-Meier analysis) were determined according to high/low TYRP1, high/low Breslow and yes/no ulceration. Cox regressions were used to calculate $P$-values, hazard ratios (HRs) and 95\% confidence intervals ( $\mathrm{Cls}$ ). ' + ' symbol indicates patients alive at the time of analysis.
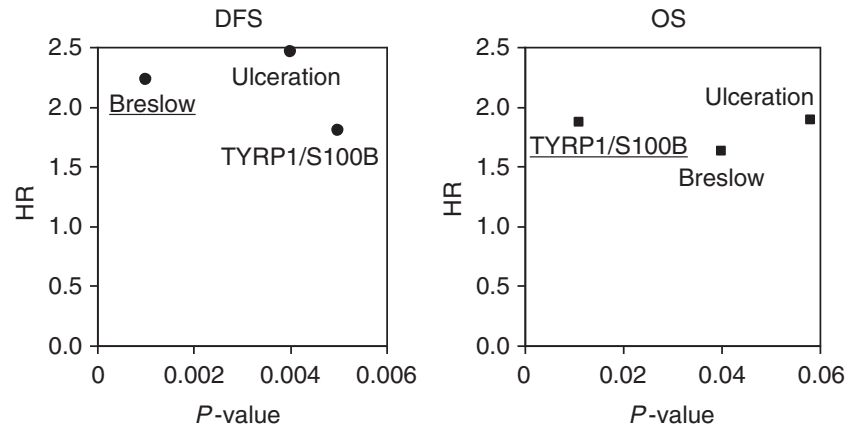

Figure 3. Comparison of the prognostic value of each of TYRP1/S100B ratio, Breslow and ulceration. P-values and hazard ratios (HRs) were calculated (Cox regression) for each prognostic markers and were compared according to DFS and OS.

metastases and Breslow/ulceration at the diagnosis showed similar predictive performances, especially for OS.

Multivariate analyses of TYRP1, Breslow and ulceration (as dichotomous variables) vs DFS and OS showed a significant 
correlation only between Breslow and DFS $(P=0.003)$. Neither TYRP1, Breslow thickness nor ulceration was significant with regard to OS. This is most probably due to the small number of residual samples in this test $(n=53,42$ events for OS) lowering significance. Indeed, univariate analysis of the same 53 samples did not show any significance neither (data not shown).

Assessment of TYRP1/S100B ratio in thin melanoma. The current study included the analyses of lymph node metastases from 10 patients with thin melanomas. As expected, we found that these patients had significantly longer DFS (median: 4.6 years) and OS (14.6 years) compared with patients with thick lesions (Breslow $>1 \mathrm{~mm}, n=84$; median DFS: 1.1 and median OS: 4.6 years, $P=0.011$ and 0.009 for DFS and OS, respectively, Mann-Whitney test). Moreover, the lymph node metastases of thin melanomas have a significant lower TYRP1/S100B ratio as compared to thick melanomas $\left(n=84\right.$; median $1.1 \times 10^{-4}$ vs $260 \times 10^{-4}, P<0.001$, Mann-Whitney test), and belonged to the group of 'low TYRP1' (ratio $<$ cutoff) associated with a better prognosis. This finding further supports the prognostic value of TYRP1/S100B and its association with Breslow.

TYRP1/S100B ratio in two recurrent lymph node metastases of the same patient. TYRP1/S100B was measured in two metastases obtained at different points in time (median: 2.1 years, range: $0.1-$ 25.8) from each of 12 patients (Figure 4). We found that the TYRP1/S100B ratio remained within the same group of poor or good prognosis for 10 out of the 12 patients. Out of these 10 patients, 3 progressed from stage III to IV without any significant impact on TYRP1/S100B ratios. This further supports that TYRP1/ $\mathrm{S} 100 \mathrm{~B}$ ratio has a prognostic value regardless of the time of metastases occurrence and suggests a well-conserved ratio during tumour progression.

\section{DISCUSSION}

Our current study aimed at evaluating the prognostic value of TYRP1/S100B mRNA expression in lymph node metastases and to compare its predictive performance to those of pathological parameters from the primary melanoma (Breslow, lymph node involvement, ulceration). We demonstrated that high TYRP1/

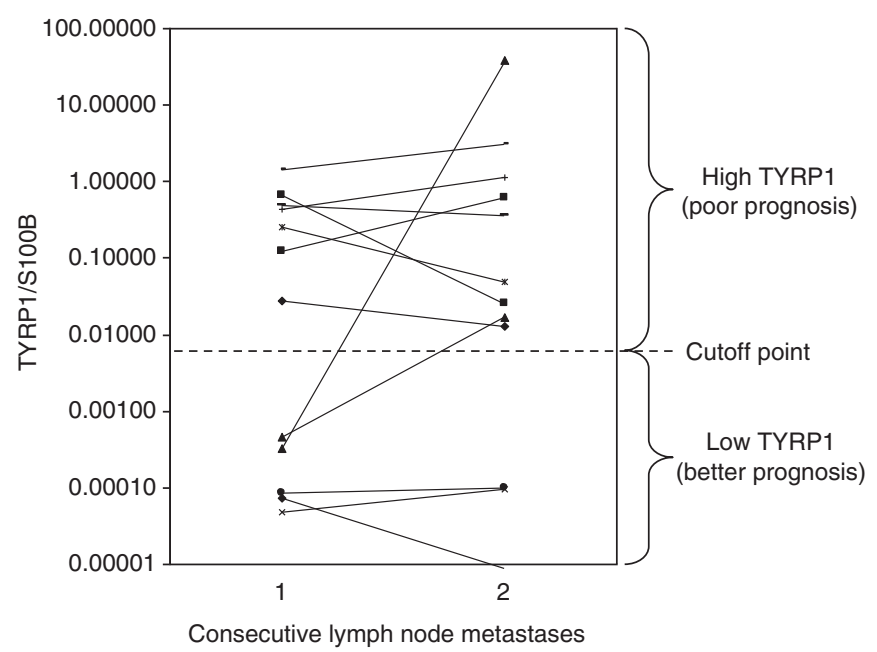

Figure 4. Variation of TYRP1/S100B ratio in two recurrent lymph node metastases within same patient. TYRP1/S100B mRNA expression were evaluated in triplicates by real-time PCR in two different melanoma lymph node metastasis obtained over years from each of 12 patients. The median TYRP1/S100B ratio calculated in Table 1 (cutoff point) is plotted.
S100B ratio significantly correlates with shorter survival (DFS and OS) as well as with high Breslow thickness and the presence of ulceration in primary lesions. Moreover, it was well conserved during melanoma progression according to high/low TYRP1 groups. Our findings, linking TYRP1 and melanoma progression, are in line with recent studies reporting that TYRP1 variants were associated with melanoma risk (Nan et al, 2009; Chatzinasiou et al, 2011).

Importantly and unlike in skin metastases, tumour cells within lymph nodes can be subcapsular, parenchymal or multifocal (Dewar et al, 2004), so that corresponding snap-frozen tissue samples are likely to contain various amounts of stroma. Of note, the wide distribution range of $S 100 \mathrm{~B} / \beta$-actin values that we observed among our samples confirms and underlines a high variation in tumour load among lymph node metastases. Therefore and to account for tumour load, we normalised TYRP1 transcript against the one of S100B. Indeed, S100B is a gene whose expression is specific of melanocyte lineage and glial cells. It has many cell functions and is mainly involved in cytoskeleton integrity, cell cycle regulation (Millward et al, 1998) and apoptosis through its interaction with p53 (Markowitz et al, 2005). Importantly, serum S100B provides a good indication of tumour burden, disease progression as well as the response to chemotherapy in stage IV melanoma patients (Hamberg et al, 2003), through a release mechanism related to cell damage or cell death (Ghanem et al, 2001). More recently, it was reported that serial determination of serum S100B in stage IIb-III melanoma patients is a strong independent prognostic marker, even stronger compared with stage and number of positive lymph nodes (Bouwhuis et al, 2011). Our data confirmed the valuable use of S100B as an indicator of tumour load (Kruijff and Hoekstra, 2012).

In our study, univariate analyses demonstrated that TYRP1/ S100B ratio $(P=0.01)$, Breslow thickness $(P=0.04)$ and ulceration $(P=0.06)$ were predictive of OS with comparable HRs (range 1.6-1.9). Unfortunately, multivariate analyses were limited by the relatively small sample size of patients with available information for all of TYRP1/S100B, Breslow and ulceration $(n=42)$. Our data are partly in accordance with a recent multivariate analysis of prognostic factors showing that the number of tumour-containing nodes, primary ulceration and patient age, but not Breslow thickness, independently predicted survival $(P<0.01)$ in a population of 268 stage III melanoma patients with nodal macrometastases (Balch et al, 2010). Of note, our population contained $18 \%$ of stage IV melanoma patients, which may impact on our observations.

It is unclear how TYRP1 expression alters cell behaviour. TYRP1 is involved in maintenance of melanosome structure and may affect melanocyte proliferation and death (Sarangarajan and Boissy, 2001). However, the link between TYRP1 and patient survival is not known yet. Actually, TYRP1 could affect melanoma progression by stabilising tyrosinase (Kobayashi et al, 1998) and protecting melanocyte against toxic melanin intermediates produced by tyrosinase ( $\mathrm{Rad}$ et al, 2004). In this context, an in vitro study from our group is on going to better understand the impact of TYRP1 on melanoma cell proliferation, migration and invasion (Mogha et al, 2011). The expression of TYRP1 is classically regulated by the microphthalmia-associated transcription factor, which is the master transcription factor involved in cell differentiation/melanogenesis, proliferation and apoptosis (Fang et al, 2002). However, additional regulatory processes could implicate microRNA. In particular, miR-155 (Li et al, 2012) and miR-137 (Dong et al, 2012) have been recently identified as downregulators of TYRP1 and microphthalmia-associated transcription factor, respectively, opening new perspectives for prognostic marker discovery. TYRP1 is also a melanocyte differentiation antigen that is important for both autoimmune destruction of melanocytes resulting in depigmentation or vitiligo, 
and anti-melanoma immune response. In this context, Liu et al (2009) reported that immunisation with a lentivector stimulated potent CD8 $\mathrm{T}$ cell responses against melanoma self-antigen TYRP1 and generated anti-tumour immunity in mice. In addition, active mouse immunisation against TYRP1-induced melanoma rejection and higher survival (Takechi et al, 1996). More recently, a new fully human anti-TYRP1 monoclonal antibody (20D7) has been developed, which recognises native TYRP1 in melanoma cells and significantly suppresses the growth of human melanoma xenografts in nude mice (Patel et al, 2007). These data allowed the activation of a phase I clinical trial that is still open using the 20D7 antibody (recently called flanvotumab, IMC-20D7S; http://clinicaltrials.gov/ ct2/show/NCT01137006).

About $3-5 \%$ of patients with thin melanoma $(\leq 1.0 \mathrm{~mm})$ develop distant metastases (Kalady et al, 2003). Additional prognostic markers could be valuable to enable more accurate stratification of such patients with regard to survival, clinical management and follow-up regimens (Murali et al, 2012). Our data showed that 10 patients with thin primary melanoma had low TYRP1/S100B mRNA expression in lymph node metastasis, which is consistent with a better prognosis, and that these patients have effectively a longer survival, as expected with a low Breslow. These observations suggest that TYRP1 marker could be also of value for patients with thin melanomas, confirming a better prognosis.

In addition, by examining 12 patients with two lymph node metastases occurring at different dates, 10 remained within the same group of high or low TYRP1/S100B. First, this supports the correlations found with DFS and OS, as all samples have been collected randomly regardless of the time of their occurrence. Second, it indicates that such ratio can be used not only as a valuable prognostic marker but also as a potential target for therapy specially that TYRP1 expression is conserved during disease progression.

In conclusion, our present study, performed in melanoma lymph node metastases, extends and confirms our previous findings in skin metastases as to a significant association between TYRP1/S100B mRNA ratio and patient survival. Its measurement in lymph node metastases of stages III and IV melanoma patients may have important clinical implications to refine initial prognosis, to establish prognosis for those with unknown or non-evaluable primaries, and possibly to impact the management of high-risk patients with TYRP1-positive lymph nodes.

\section{ACKNOWLEDGEMENTS}

This study received financial support from MEDIC Foundation, Les Amis de l'Institut Bordet, Fondation Lambeau-Marteaux, EORTC-Melanoma Group and Wallonie-Bruxelles International. Petra El Hajj is the recipient of a fellowship from the Lebanese National Council for Scientific Research and the Lebanese University.

\section{REFERENCES}

Alonzo TA (2005) Standards for reporting prognostic tumor marker studies. J Clin Oncol 23: 9053-9054.

Balch CM, Gershenwald JE, Soong S-J, Thompson JF, Atkins MB, Byrd DR, Buzaid AC, Cochran AJ, Coit DG, Ding S, Eggermont AM, Flaherty KT, Gimotty PA, Kirkwood JM, McMasters KM, Mihm Jr MC, Morton DL, Ross MI, Sober AJ, Sondak VK (2009) Final version of 2009 AJCC melanoma staging and classification. J Clin Oncol 27: 6199-6206.

Balch CM, Gershenwald JE, Soong S-J, Thompson JF, Ding S, Byrd DR, Cascinelli N, Cochran AJ, Coit DG, Eggermont AM, Johnson T, Kirkwood JM, Leong SP, McMasters KM, Mihm Jr MC, Morton DL, Ross MI, Sondak VK (2010) Multivariate analysis of prognostic factors among 2,313 patients with stage III melanoma: comparison of nodal micrometastases versus macrometastases. J Clin Oncol 28: 2452-2459.

Berger AJ, Kluger HM, Li N, Kielhorn E, Halaban R, Ronai Z, Rimm DL (2003) Subcellular localization of activating transcription factor 2 in melanoma specimens predicts patient survival. Cancer Res 63: 8103-8107.

Berwick M, Erdei E, Hay J (2009) Melanoma epidemiology and public health. Dermatol Clin 27: 205-214viii.

Bouwhuis MG, Suciu S, Kruit W, Salès F, Stoitchkov K, Patel P, Cocquyt V, Thomas J, Liénard D, Eggermont AMM, Ghanem G (2011) Prognostic value of serial blood S100B determinations in stage IIB-III melanoma patients: a corollary study to EORTC trial 18952. Eur J Cancer 47: 361-368.

Chatzinasiou F, Lill CM, Kypreou K, Stefanaki I, Nicolaou V, Spyrou G, Evangelou E, Roehr JT, Kodela E, Katsambas A, Tsao H, Ioannidis JPA, Bertram L, Stratigos AJ (2011) Comprehensive field synopsis and systematic meta-analyses of genetic association studies in cutaneous melanoma. J Natl Cancer Inst 103: 1227-1235.

Chen G, Cheng Y, Zhang Z, Martinka M, Li G (2011) Prognostic significance of cytoplasmic p27 expression in human melanoma. Cancer Epidemiol Biomarkers Prev 20: 2212-2221.

Dewar DJ, Newell B, Green MA, Topping AP, Powell BWe, Cook MG (2004) The microanatomic location of metastatic melanoma in sentinel lymph nodes predicts nonsentinel lymph node involvement. J Clin Oncol 22: 3345-3349.

Dong C, Wang H, Xue L, Dong Y, Yang L, Fan R, Yu X, Tian X, Ma S, Smith GW (2012) Coat color determination by miR-137 mediated down-regulation of microphthalmia-associated transcription factor in a mouse model. RNA 18: 1679-1686.

Díaz-Lagares A, Alegre E, Arroyo A, González-Cao M, Zudaire ME, Viteri S, Martín-Algarra S, González A (2011) Evaluation of multiple serum markers in advanced melanoma. Tumour Biol 32: 1155-1161.

Fang D, Tsuji Y, Setaluri V (2002) Selective down-regulation of tyrosinase family gene TYRP1 by inhibition of the activity of melanocyte transcription factor, MITF. Nucleic Acids Res 30: 3096-3106.

Ghanem G, Loir B, Morandini R, Sales F, Lienard D, Eggermont A, Lejeune F (2001) On the release and half-life of S100B protein in the peripheral blood of melanoma patients. Int J Cancer 94: 586-590.

Hamberg AP, Korse CM, Bonfrer JM, de Gast GC (2003) Serum S100B is suitable for prediction and monitoring of response to chemoimmunotherapy in metastatic malignant melanoma. Melanoma Res 13: 45-49.

Henry L, Lavabre-Bertrand T, Douche T, Uttenweiler-Joseph S, Fabbro-Peray P, Monsarrat B, Martinez J, Meunier L, Stoebner P-E (2010) Diagnostic value and prognostic significance of plasmatic proteasome level in patients with melanoma. Exp Dermatol 19: 1054-1059.

Journe F, Id Boufker H, Van Kempen L, Galibert M-D, Wiedig M, Salès F, Theunis A, Nonclercq D, Frau A, Laurent G, Awada A, Ghanem G (2011) TYRP1 mRNA expression in melanoma metastases correlates with clinical outcome. Br J Cancer 105: 1726-1732.

Kalady MF, White RR, Johnson JL, Tyler DS, Seigler HF (2003) Thin melanomas: predictive lethal characteristics from a 30 -year clinical experience. Ann Surg 238: 528-535discussion 535-537.

Kobayashi T, Imokawa G, Bennett DC, Hearing VJ (1998) Tyrosinase stabilization by Tyrp1 (the brown locus protein). J Biol Chem 273: 31801-31805.

Kruijff S, Hoekstra HJ (2012) The current status of S-100B as a biomarker in melanoma. Eur J Surg Oncol 38: 281-285.

Lazova R, Camp RL, Klump V, Siddiqui SF, Amaravadi RK, Pawelek JM (2012) Punctate LC3B expression is a common feature of solid tumors and associated with proliferation, metastasis, and poor outcome. Clin Cancer Res 18: 370-379.

Li J, Liu Y, Xin X, Kim TS, Cabeza EA, Ren J, Nielsen R, Wrana JL, Zhang Z (2012) Evidence for positive selection on a number of microRNA regulatory interactions during recent human evolution. PLoS Genet $\mathbf{8}$ : e1002578.

Liu Y, Peng Y, Mi M, Guevara-Patino J, Munn DH, Fu N, He Y (2009) Lentivector immunization stimulates potent CD8 $\mathrm{T}$ cell responses against melanoma self-antigen tyrosinase-related protein 1 and generates antitumor immunity in mice. J Immunol 182: 5960-5969.

Mandruzzato S, Callegaro A, Turcatel G, Francescato S, Montesco MC, Chiarion-Sileni V, Mocellin S, Rossi CR, Bicciato S, Wang E, Marincola FM, Zanovello P (2006) A gene expression signature associated with survival in metastatic melanoma. J Transl Med 4: 50.

Markowitz J, Mackerell Jr AD, Carrier F, Charpentier TH, Weber DJ (2005) Design of Inhibitors for S100B. Curr Top Med Chem 5: 1093-1108. 
McShane LM, Altman DG, Sauerbrei W, Taube SE, Gion M, Clark GM (2005) REporting recommendations for tumour MARKer prognostic studies (REMARK). Br J Cancer 93: 387-391.

Miller AJ, Mihm Jr MC (2006) Melanoma. N Engl J Med 355: 51-65.

Millward TA, Heizmann CW, Schäfer BW, Hemmings BA (1998) Calcium regulation of $\mathrm{Ndr}$ protein kinase mediated by $\mathrm{S} 100$ calcium-binding proteins. EMBO J 17: 5913-5922.

Mogha A, Gilot D, Primot A, Debbache J, Journe F, Bennett DC, Dreno B, Napolitano A, Ghanem G, Galiber MD (2011) Uncovered role of Tyrosinase-related Protein 1 (TYRP1) in melanoma cells aggressiveness. Pig Cell Mel Res 24: 791 abstract C44.

Mouawad R, Spano J-P, Comperat E, Capron F, Khayat D (2009) Tumoural expression and circulating level of VEGFR-3 (Flt-4) in metastatic melanoma patients: correlation with clinical parameters and outcome. Eur J Cancer 45: 1407-1414.

Murali R, Haydu LE, Long GV, Quinn MJ, Saw RPM, Shannon K, Spillane AJ, Stretch JR, Kefford RF, Thompson JF, Scolyer RA (2012) Clinical and pathologic factors associated with distant metastasis and survival in patients with thin primary cutaneous melanoma. Ann Surg Oncol 19 1782-1789.

Nan H, Kraft P, Hunter DJ, Han J (2009) Genetic variants in pigmentation genes, pigmentary phenotypes, and risk of skin cancer in Caucasians. Int $J$ Cancer 125: 909-917.

Patel D, Balderes P, Lahiji A, Melchior M, Ng S, Bassi R, Wu Y, Griffith H, Jimenez X, Ludwig DL, Hicklin DJ, Kang X (2007) Generation and characterization of a therapeutic human antibody to melanoma antigen TYRP1. Hum Antibodies 16: 127-136.

Rad HH, Yamashita T, Jin H-Y, Hirosaki K, Wakamatsu K, Ito S, Jimbow K (2004) Tyrosinase-related proteins suppress tyrosinase-mediated cell death of melanocytes and melanoma cells. Exp Cell Res 298: 317-328.

Sarangarajan R, Boissy RE (2001) Tyrp1 and oculocutaneous albinism type 3. Pigment Cell Res 14: 437-444.

Stoitchkov K, Letellier S, Garnier J-P, Bousquet B, Tsankov N, Morel P, Ghanem G, Le Bricon T (2003) Evaluation of the serum L-dopa/L-tyrosine ratio as a melanoma marker. Melanoma Res 13: 587-593.

Takechi Y, Hara I, Naftzger C, Xu Y, Houghton AN (1996) A melanosomal membrane protein is a cell surface target for melanoma therapy. Clin Cancer Res 2: 1837-1842.

van Houdt IS, Oudejans JJ, van den Eertwegh AJM, Baars A, Vos W, Bladergroen BA, Rimoldi D, Muris JJF, Hooijberg E, Gundy CM, Meijer CJLM, Kummer JA (2005) Expression of the apoptosis inhibitor protease inhibitor 9 predicts clinical outcome in vaccinated patients with stage III and IV melanoma. Clin Cancer Res 11: 6400-6407.

This work is published under the standard license to publish agreement. After 12 months the work will become freely available and the license terms will switch to a Creative Commons AttributionNonCommercial-Share Alike 3.0 Unported License. 